\title{
Novel non-invasive Adjunctive Techniques for Early Oral Cancer Diagnosis and Oral Lesions Examination
}

\author{
Valeria Mercadante ${ }^{\mathrm{a}, *}$, Carlo Paderni $^{\mathrm{b}}$ and Giuseppina Campisi ${ }^{\mathrm{b}}$ \\ ${ }^{a}$ University College London Eastman Dental Institute, London, UK; ${ }^{b}$ Sector of Oral Medicine “V. Margiotta”, Section of Oral Sci- \\ ences, Department of Surgical and Oncological Disciplines, University of Palermo, Palermo, Italy
}

\begin{abstract}
Oral cancer is a potentially fatal disease with an increasing incidence and an unchanged 5-year mortality rate. Unfortunately, oral cancer is often still late diagnosed, which leads to an increase in the likelihood of functional impairment due to treatment and mortality rate. Definitive diagnosis of oral cancer must be confirmed by scalpel biopsy and histological assessment. However despite its benefits, scalpel biopsy is invasive and it is burdened by a potential morbidity. Furthermore, previous studies have suggested a high degree of intraobserver and interobserver variability regarding the histological evaluation of malignancy. As a consequence, in recent years there has been a growing and persisting demand towards developing new non-invasive, practical diagnostic tools that might facilitate the early detection of oral cancer. The most investigated non-invasive adjunctive techniques are vital staining, autofluorescence, chemiluminescence, narrow band imaging, and exfoliative cytology. Aim of the review is to critically describe these adjunctive aids and, after considering the literature data, an expert opinion on the effectiveness and the possible use of each technique will be provided.
\end{abstract}

Keywords: Oral cancer, potentially malignant disorder, early diagnosis, screening, vital staining, autofluorescence, chemiluminescence, narrow band imaging, exfoliative cytology, oral biopsy.

\section{INTRODUCTION}

Oral cancer is the sixth most common cancer worldwide with increasing incidence and mortality rate [1-3]. Its early detection is crucial to improve survival and reduce morbidity, disfigurement, loss of function, poor quality of life, duration of treatment and hospital costs $[4,5]$. Despite numerous technological advances over the last fifty years, the survival rate linked to Oral Squamous Cell Carcinoma (OSCC) did not undergo a marked improvement, and it amounts to approximately $50 \%$ at five years [6]. The poor prognosis is due to several factors, including the advanced stage of disease (Stage III or IV) at diagnosis. Despite the oral cavity is a readily accessible site for a visual examination and palpation and the nature of the neoplastic process which is considered as a multi-stage phenomenon, often evolving from oral Potentially Malignant Disorders (PMDs), OSCCs are still late diagnosed [7]. The 5-year survival rate for patients with primary oral cancer is still one of the lowest between the most invasive cancers, with loco-regional recurrence as primary cause of death [8]. Consequently, an intensive follow-up regimen is set up to discover recurrences or a second primary cancer as early as possible. OSCC stage at the time of diagnosis influences also the therapeutic options available with significant risk of acute and chronic side effects above all related to surgical/nonsurgical cancer therapy, reducing patient quality of life and the likelihood of long term survival [9].

The problem of the diagnostic delay could be partly solved by using two different approaches: first, screening programs to identify asymptomatic patients with suspected "high risk" lesions and then, improving the ability to detect early cancer lesions and to identify the PMDs having an increased risk of malignant transformation. To address these issues, several practical guides have been published and specific diagnostic tools have been developed to identify dysplasia and cancer lesions in asymptomatic patients with an oral abnormality.

*Address correspondence to this author at Oral Medicine Unit, Division of Maxillofacial, Diagnostic, Medical and Surgical Sciences, Eastman Dental Institute, University College London, 256 Gray's Inn Road, London WC1X 8LD, UK; Tel: +44 (0)2034561182;

E-mail: valeria.mercadante.11@ucl.ac.uk
The diagnostic process begins with a comprehensive medical history and a head and neck examination. Dentists are professionally responsible for providing a comprehensive oral examination and follow-up for their patients: an early diagnosis of oral cancer could mean the difference between life and death. The Conventional Oral Exam (COE) under white light is a non-invasive and simple procedure whose goal is to detect any nodule, swelling, mucosal alteration (ulcerations, textural or colour changes) and/or unexplained neck lymph nodal adenopathy. COE has been considered as a life-saving test [10], which seems to be associated with a reduction in mortality among high-risk patients, especially when performed by highly qualified staff [11]. However, there is evidence that the COE alone may be of limited value as a method of screening for oral malignancies $[12,13]$. On the contrary, a relatively high rate of sensitivity and specificity for COE has been reported [14]. Although the clinical examination has been increasingly used in the past and it has been considered as the first step of the diagnostic process in order to define a provisional diagnosis, which may be confirmed by further investigations, COE is strongly dependent on the experience in oral medicine of the clinician who performs it. The classic presentation of a potentially malignant disorder or early cancer lesions as a red or white lesion, persistent ulcer, provides some difficulties in the differential diagnosis with other benign conditions. In the presence of these lesions, COE is unable to identify those at "high risk" of progression. Furthermore, recent studies suggest that dysplasia or micro-invasive carcinoma can occur in clinically normal-appearing mucosa [15]. The clinical examination is absolutely useful in the discovery of oral lesions, but it seems not be able to identify accurately the biologically relevant lesions susceptible to neoplastic transformation. Currently, the gold standard diagnostic test for OSCC is an oral biopsy with histopathology. Unfortunately, available studies reported inter-observed and interobserver variability in the histologic diagnosis of dysplasia and SCC, with reported agreement rate of only $56 \%$ [16]. Clinical features of the lesion, specimen characteristics, artefacts resulting from crushing, fulguration or incorrect fixation and freezing, experience of the pathologist may play a part in determining the diagnosis $[17,18]$. The grade of malignancy and depth of invasion are also important prognostic factors, strongly influenced by the size and the depth of biopsy [19-21]. 
This finding suggests the need to reduce the variability in the diagnosis of oral malignant lesions. This goal can be achieved implementing techniques to early detect malignant alterations in the oral cavity.

However, there is a wide range of available tools that could be used in order to identify early signs of cancer. With so many commercial diagnostic aids and adjunctive techniques to choose from these days, it becomes hard for the dentist to determine if a diagnostic tool will be appropriate and useful for oral cancer screening.

Aim of the present paper is to present available evidence, if present, regarding the adjunctive techniques that may facilitate the early detection of oral cancer, citing and discussing also systematic review and meta-analysis on this topic. The diagnostic adjunctive techniques evaluated in this review are vital staining, chemiluminescence, direct autofluorescence, exfoliative cytology and narrowband imaging.

\section{VITAL STAINING}

The vital staining is a technique that exploits the properties of certain dyes to highlight some tissues, cells or cellular organelles. Available studies reported that the examination of the oral mucosa can be performed by staining with rose Bengal, already widely used to diagnose various ocular surface disorders [22, 23], methylene blue, recently proposed for screening gastrointestinal or prostate tumours because it seems less toxic to the human [24, 25] and Lugol's solution, used to detection esophageal, gastrointestinal and gynecological abnormalities [26-28]. However, the more common vital stain in oral medicine is the toluidine blue (TB), a vital acidophilic metachromatic stain of the thiazine group that has been effectively used in nuclear staining because of its binding to DNA nucleic acid. For this reason, it has been widely used to facilitate early detection of malignant lesions and by the surgeons to demarcate intra-operatively the extension of the lesion [29]. In recent years, the diagnostic ability of the TB staining was evaluated in a large number of studies. Epstein et al. showed the increase of the sensitivity of clinical examination after the application of TB in the detection of malignant lesions in high-risk patients [30]. There are some evidences that TB appears to improve precancerous lesion visualization by showing high-risk areas (areas of high cell proliferation), therefore guiding biopsy. In vivo, toluidine blue stains deoxyribonucleic acid and may be retained in intracellular spaces of dysplastic [31]. Furthermore, it can be found in dysplastic cells and malignant tissues at sites of loss of tumour suppressor genes, potentially predicting a malignant transformation of premalignant lesion or representing a OSCC at diagnosis [32]. Some Authors suggest also that the staining intensity after TB application may provide important information related to the binding of the stain with the molecular changes, predicting the neoplastic risk, most importantly in case of second OSCC or recurrence [33]. In another study, the Authors investigated the molecular profile of PMDs positive and negative after TB staining, by evaluating the relationship between intensity of staining and chromosomal aberrations [34]. The loss of heterozygosis, which occurs more frequently in samples positive to $\mathrm{TB}$, may indicate an increased risk of neoplastic progression [35]. Zhang et al. showed a progression risk four times higher in lesions with mild dysplasia or benign histopathology, when they were positive to TB [33].

The analysis of the current scientific evidence suggests that the sensitivity is higher in cancer cases than in cases of dysplasia. Overall, the sensitivity of this technique for detection of oral cancer range from 0.78 to 1.00 and specificity from 0.31 to 1.00 [36]. Although TB is highly sensitive and moderately specific for malignant lesions, it is far less sensitive to PMDs with false-negative rates of up to 58\% reported for identifying mild to moderate dysplasia [37].

False negatives occur in a minority of cases, especially in the most recent case series in which the product has undergone some improvements. Nevertheless, the binding of TB to the nucleic acid may occur also in the mucosal ulceration, granulation tissue and inflammatory lesions thereby contributing to the number of false positives. However, unlike in the malignant lesions, the coloration of these traumatic benign lesions does not persist for a long time and is often located on the periphery of ulceration. To reduce the number of inflammatory lesions that can cause false positives, it is highly recommended a further assessment of non-healing wound after 15 days at least.

There is evidence that the TB test is helpful in identifying oral cancers, confirmed by the positive clinical experience of clinicians that have used this approach for years. Nevertheless, there is insufficient evidence to recommend for or against the stand-alone use of TB to enhance the identification of potentially malignant lesions in the general population. [38]. However, considering the dramatic implications of undiagnosed OSCC, and being the application of toluidine blue staining fast, minimally invasive, inexpensive and well patient-accepted, in this review the Authors would support the use of TB as a diagnostic tools in the hands of clinicians adequately trained in the exact interpretation of its results (Fig. 1a-b). It is also strongly recommended not using it as a substitute for biopsy, but rather as a complementary aid during the diagnostic phase, guiding the choice of the biopsy correct site and in the follow-up phase in order to early detect any recurrence or secondary OSCC.

\section{CHEMILUMINESCENCE}

The term "chemiluminescence" refers to the emission of light radiation in the visible range after the electrons, excited by a chemical exergonic reaction, returning from the excited to the ground state; the potential energy of electronic transitions within the atoms or molecules is thus released in the form of light photons. This technique is correctly based on the reflectance phenomenon that indicates the proportion of incident light that a given surface is able to reflect. This technique has been used for many years as a diagnostic aid in the examination of cervical mucosa for the detection of potentially or malignant lesions.

Dehydration with acetic acid highlights the altered nuclearcytoplasmic ratio of the neoplastic epithelial cells and thus malignant tissues assume a characteristic "aceto-white" appearance [3941]. This phenomenon can be further amplified by replacing conventional lighting with diffuse blue-white chemiluminescent illumination.

Considering the morphological similarities between cervical and oral epithelia, recently this technique has been proposed also for the oral cavity [42]. Approved by the FDA and introduced into the market under the name of ViziLite Plus ${ }^{\circledR}$ (Zila Pharmaceuticals, Fort Collins, USA), Microlux DL ${ }^{\circledR}$ (AdDent, Danbury, USA) and Orascoptic DK ${ }^{\mathrm{TM}}$ (Orascoptic, Middleton, USA), these diagnostic aids aim to enable a better identification, assessment and monitoring of oral mucosal abnormalities in populations at increased risk of oral cancer. The main difference between the available disposals is that ViziLite Plus involves the use of single-use chemiluminescent stick, while Microlux DL and Orascoptic DX provide a blue-white LED fiber optic light.

ViziLite Plus ${ }^{\circledR}$ consists of the emission of light from a chemical reaction between hydrogen peroxide and acetylsalicylic acid inside a capsule stick. During the chemical reaction, is produced a light white-blue with a wavelength between $430 \mathrm{~nm}$ and $580 \mathrm{~nm}$ that lasts for around $10 \mathrm{~min}$. The first step of the procedure includes a first intra-oral examination under conventional white light in order to evaluate the location, extent, margins, presence of satellite lesions and visibility. Then, the intraoral exploration is repeated by using ViziLite light, after a $60-\mathrm{sec} 1 \%$ acid acetic rinse in order to remove the glycoprotein barrier and slightly dry the oral mucosa. Under chemiluminescent light, the healthy tissue, able to absorb it, appears dark blue, while the abnormal tissue, characterized by excessive keratinization, increase in the ratio nucleus/cytoplasm, and 

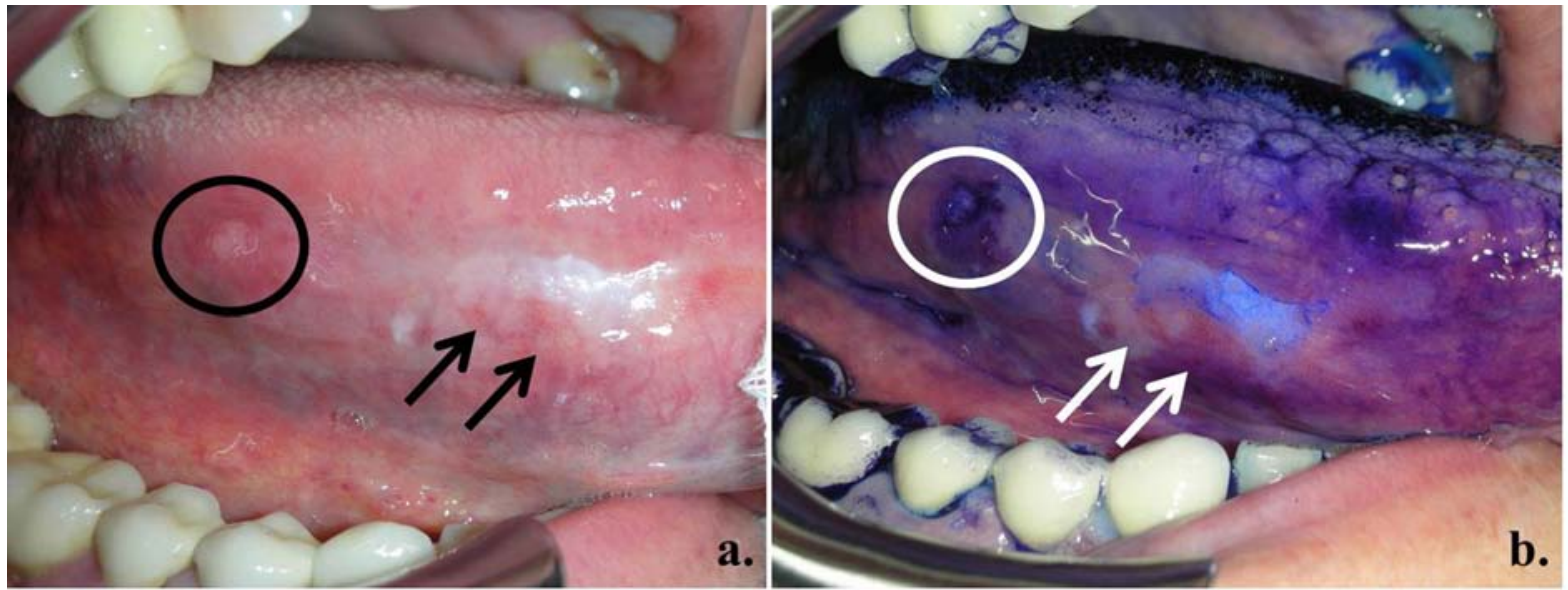

Fig. (1a-b). a) Oral leukoplakia on the right margin of the tongue is showed (black arrows). Note the presence of an erythro-leucoplakia lesion on the posterior third of the lingual border (black circle). b) It shows the same lesion after TB staining. The vital dye is strongly picked up by the posterior lesion (white circle) and does not stain the anterior lesion, where it is most evident the presence of red areas within the white lesion, thanks to the increase of contrast (white arrows). After TB staining, a biopsy has been performed at both sites. The posterior lesion revealed to be histologically an erythroplakia with severe dysplasia, while the anterior lesion, no TB positive, was found to be a leukoplakia in the absence of dysplasia. (The color version of the figure is available in the electronic copy of the article).

chronic inflammatory infiltrate, reflect the reflect the light and appears white (aceto-white lesion) with distinct margins [43, 44].

Many studies have examined the reflectance of the tissue after rinsing with acetic acid as an aid to screening for oral cancer [36]. Although ViziLite is widely used in the United States for the evaluation of suspicious oral lesions, especially when used in association with the Toluidine Blue solution to mark the lesions to posterior biopsy, evidence is lacking in support of its effectiveness. A recent review [45] reported that sensitivity of this device was $100 \%$ and specificity ranged from $0 \%-14.2 \%$, showing that this method is not able to discern between benign hyperkeratosis, inflammatory diseases, OSCC and PMDs [46-49]. Moreover, the reported high sensitivity could be related to the design of the studies, which often involved only patients with mucosal lesions previously visualized under conventional light. In short, the evidence supporting the use of the system to aid the early detection of OSCC and PMDs is actually quite scarce.

However, available evidence suggests the use of chemiluminescence to enhance visual parameters of the lesions in terms of brightness, sharpness (margin delineation), surface texture and, in some cases, size of lesion compared with parameters after conventional light. Due to the improved visual parameters, chemiluminescence-based device can be considered a useful tool for investigating oral mucosa and improving the visualization of white lesions [50], but does not increase the early detection of PMDs and is not recognized an additional clinical benefit, since the additional costs that arise from its use [51, 52].

The above-mentioned Microlux/DL ${ }^{\circledR}$, which shares the basic principles of ViziLite, consists of a light-emitting diode (Power LED), a reusable light source that produces a diffused light. A recent study evaluated the sensitivity and specificity of Microlux/DL, showing values of $77.8 \%$ and $70.7 \%$ respectively, although it is a poor discriminator for inflammatory, traumatic, and malignant lesions.

Available studies reported that this approach does not seem to be significantly more useful than a clinical examination under conventional LED white-light [53] (Fig. 2a-b).

Further controlled trials are needed to determine specifically the ability of these devices in early detecting PMDs clinically not visible under conventional light.
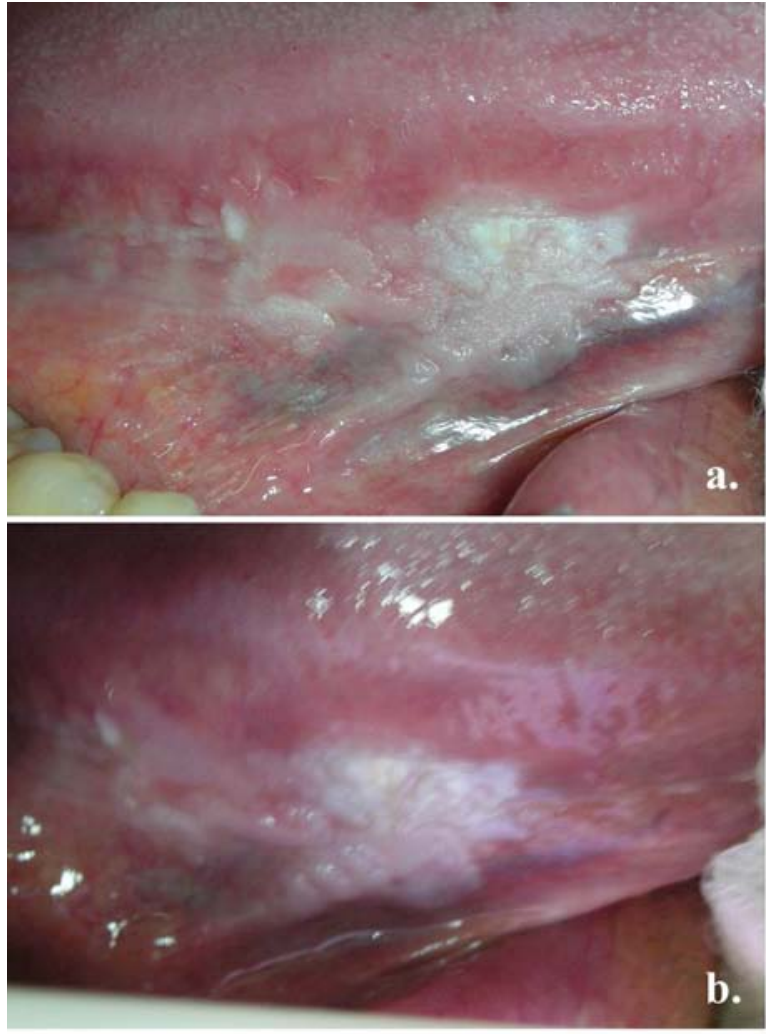

Fig. (2a-b). a) Non homogenous leukoplakia on right lingual margin after clinical examination under conventional incandescent light; b) appearance of the lesion after clinical exam under chemiluminescent light. The technique did not result in any improvement of the lesion visualization.

\section{DIRECT AUTOFLUORESCENCE IMAGING}

The direct autofluorescence is a method that exploits the ability of some molecules (fluorophores) to absorb a light of a particular wavelength and release it with a greater wavelength after short time. Normal healthy cells contain endogenous fluorophores such as Nicotinamide Adenine Dinucleotide (NADH), Flavin Adenine 
Dinucleotide (FAD) and the cross-linked collagen, which show broad excitation spectra $(250-450 \mathrm{~nm})$ and release $500 \mathrm{~nm}$ light when subjected to a blue-violet light (400-460 nm), appearing as a green-apple areas. It has been demonstrated that changes in the structure and metabolism of the mucosae alter the distribution of fluorophores within the tissue [36].

Abnormal cells have been shown not able to reflect fluorescent light, and thus absorb it, appearing as dark areas under 400-460 nm light.

Recent studies have shown that the autofluorescence imaging represents an incredibly promising tool in screening and diagnosis of premalignant conditions of the lung, cervix, skin, and finally the mouth [54-57]. The mechanism behind tissue autofluorescence tissue has been extensively described elsewhere [58, 59]. Briefly, loss of autofluorescence is believed to reflect the complex and progressive morphological and biochemical changes, typical of squamous epithelial carcinogenesis [60]. During cancer progression, the optical properties of epithelial surface and underlying stroma are altered, due to changes in structure (e.g. hyperkeratosis, hyperchromatin and increased cellular/nuclear pleomorphism) and metabolism (concentration of FAD and NADH within the epithelium, as well as changes of the sub-epithelial stroma in terms of composition of the collagen matrix and elastin) [61]. These changes can alter the distribution of tissue fluorophores and as a consequence, an increased light absorption or diffusion after stimulation with a blue (400-460 nm) light can be observed, which in turn reduces and modifies the detectable autofluorescence.

Several studies have evaluated the possibility of using tissue autofluorescence to differentiate and early detect cancers of the oral cavity $[62,63]$. Commercially available diagnostic tools based on direct autofluorescence imaging are VELscope ${ }^{\circledR}$ (LED Dental, Vancouver, British Columbia, Canada) and Sapphire ${ }^{\circledR}$ Plus (DenMat Holdings, Santa Maria, CA). These tools consist of portable devices developed in order to improve the detection capability of OSCC and PMDs [64, 65], by highlighting the loss of tissue fluorescence correlated with some oral diseases, thanks the use of a 400-460 nm light with a wavelength compatible with the emission spectrum of the endogenous fluorophores [66].

This technique involves first a clinical examination under conventional white light and, after turning off the lights, a further clinical examination by using the handpiece device, keeping at a distance of about $5 \mathrm{~cm}$ from the oral cavity to optimize the visualization of tissue fluorescence.

In the healthy oral cavity, there are components that may positively or negatively affect the tissue fluorescence. Some components such as the blood hemoglobin and melanin have been shown able to reduce fluorescence, while fibrin, keratin and porphyrin appear to increase the fluorescence.

For this reason, a pattern of fluorescence within the oral mucosa can be normally described, depending on the oral site. For example, the fungiform papillae on the tongue dorsal surface appear as dark areas, since the end of filiform papillae are keratinized and emits a fluorescent light; along the tongue ventral surface, blood vessels appear dark, because of the high blood flow and finally the attached gingiva is often clear for its ultrastructural characteristics, being rich in keratin.

In addition, the oral cavity is naturally exposed to various degrees of mild inflammation and chronic irritation, and thus some oral sites could show a darker appearance characterized by poorly defined boundaries, caused by the increased inflow of blood.

If a dark area appear during direct fluorescence visualization, the oral lesion must be considered as a suspected one and the clinical examination should be repeated by applying a pressure with the back of a dental mirror or a similar tool with a sweeping motion, in order to remove blood from the examined area. If the normal apple- green fluorescence returns after this pressure, the lesion is likely to have an inflammatory component (Fig. 3a-b).

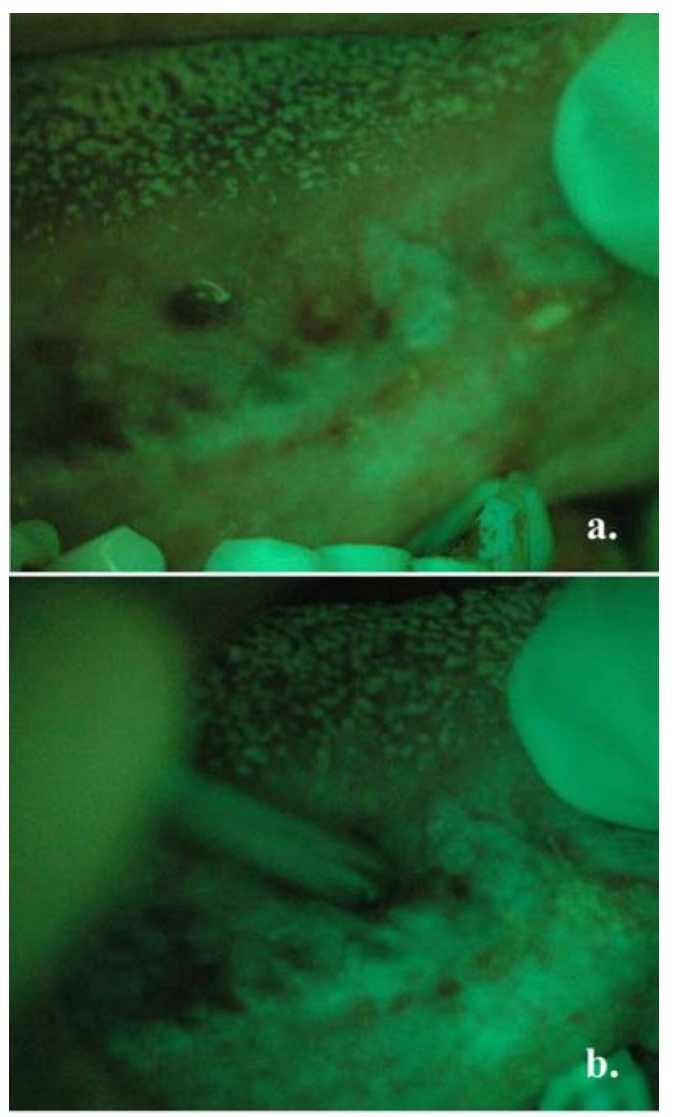

Fig. (3a-b). a) The figure shows a white lesion on the lingual right margin. In addition, it shows the presence of dark areas near to the white lesion; $\mathbf{b}$ ) considering the lesion location and its clinical features being compatible with lingual varices, the compression of the dark area with subsequent reappearance of tissue fluorescence has been performed.

Available evidence suggests that this technique can facilitate the detection of neoplastic lesions, with value of sensitivity and specificity ranging from $18.4 \%$ to $98 \%$ and from $81 \%$ to $100 \%$, respectively [67-70]. Some Authors reported that autofluorescencebased device can identify lesions which were not clinically evident $[71,72]$, as well as high risk areas for malignant transformation [71]. Limitations of autofluorescence imaging in discriminating between dysplastic and non-dysplastic have been reported in a recent study [73], although another study showed that the device achieves a sensitivity of $98 \%$ and specificity of $100 \%$ when discriminating normal mucosa from severe dysplasia/carcinoma in situ (CIS) or invasive carcinoma [66].

However, there is no full agree in literature regarding the ability of a autofluorescence-based device to distinguish between OSCC and other oral diseases such as aphthous ulcers, lichen planus and pemphigoid [74]. In a recent study, Poh et al. demonstrated that the direct tissue fluorescence visualization can enhance the delineation of the neoplastic margins [75]. In some cases, the loss of fluorescence was extended beyond $25 \mathrm{~mm}$ from the clinically visible (under WL) margins of the lesion. This is extremely interesting, considering that one of the most difficult and contentious issues, about the treatment of OSCC, involves the decision concerning the amount of the surrounding normal-looking tissues that have to be removed in addition to the tumour mass. Generally, surgeons arbitrarily extend $10 \mathrm{~mm}$ or more beyond the margins of a neoplastic lesion, in order to remove any clinically occult high-risk areas [76]. 
Unfortunately, this approach often fails because neoplastic or dysplastic areas often can be detected in surgical margins [77]. Therefore, the feasible use of tissue fluorescence as a guide for the detection of field alterations and the extension of tumour margins during surgical excision is extremely interesting, but still a number of efforts should be performed to validate this hypothesis.

There is also evidence of significant clinical value of this device in a referral clinic specialized in diagnosing and treating oral cancer [78]. This non-invasive tool should be seen as complementary device that cannot fully replace the histopathological assessment during diagnosis and monitoring steps (Fig. 4a-c). During the diagnostic moment, such device could enhance the visualization of clinical features of lesions and in particular, it could help clinicians in selecting the correct site or sites to be submitted to biopsy, thanks to highlighting the margins. During the follow-up step, this tool could be useful, especially within an oral medicine secondary care facility, in clinical monitoring of OPMDs with definite histological diagnosis and in patients with a previous history of OSCC $[79,80]$.

\section{EXFOLIATIVE CYTOLOGY}

Exfoliative cytology consists in collecting cell samples from mucosal surfaces by means of scraping, brushing or rinse in order to detect any cytological alteration. This technique was first designed for cervical cancer early detection [19] and it has recently proposed in oral medicine to detect primitive cell changes related to malignancy. This technique is also useful for the diagnosis of oral lesions related to viral and fungal diseases [81]. However, the main limitation of this method is the collection of disaggregated cells, which therefore does not allow the pathologist to reach a definitive diagnosis. Available studies reported that sensitivity and specificity of conventional exfoliative cytology in detecting OSCC lesions, ranged between $76.8 \%-100 \%$, and $88.9 \%-100 \%$, respectively [82]. Brushing proved to be a more convenient collecting method compared with the wooden spatula when dealing with oral lesions [83]. The combined use of TB staining and brush cytology was found to be highly sensitive and moderately specific for OSCC lesions (6\% false-negative rate) but less sensitive to the PMD lesions with a sensitivity of $89 \%$ and a specificity of $92 \%$ [37].

Liquid-Based Cytology (LBC) is a sample filtration method originally developed to provide a near-monolayer of superficial cervical cells, which can be more easily inspected. A study reported that the application of LBC on oral cell samples collected by brushing can significant improve cell distribution and smear thickness, leading to an easier identification of abnormal cells and reporting an higher sensitivity and specificity $(95.1 \%$ and $99.0 \%$ respectively) [84]. However, the LBC method was designed just for superficial cytology samples, thus it leads to the destruction of epithelial fragments, so it is likely that the use of LBC for diagnosing oral lesions is of minimal value [51].

Recently, a new technique has been developed to improve the reliability of this method in investigating mucosal lesions not subjected to biopsy. OralCDx ${ }^{\circledR}$ (OralScan Laboratories, Inc., Suffern, $\mathrm{NY}$ ) is a computer-assisted method for the analysis of cellular samples collected by using a patented brush. This technique method, unlike the conventional cytology, allows obtaining a complete transepithelial sample in which all epithelial layers (superficial, intermediate and basal) are represented. The full-thickness epithelium sampling is essential for a correct diagnosis [85].

The sampling is obtained from a brush specifically designed to improve the inadequate samples of standard cytology, which re-
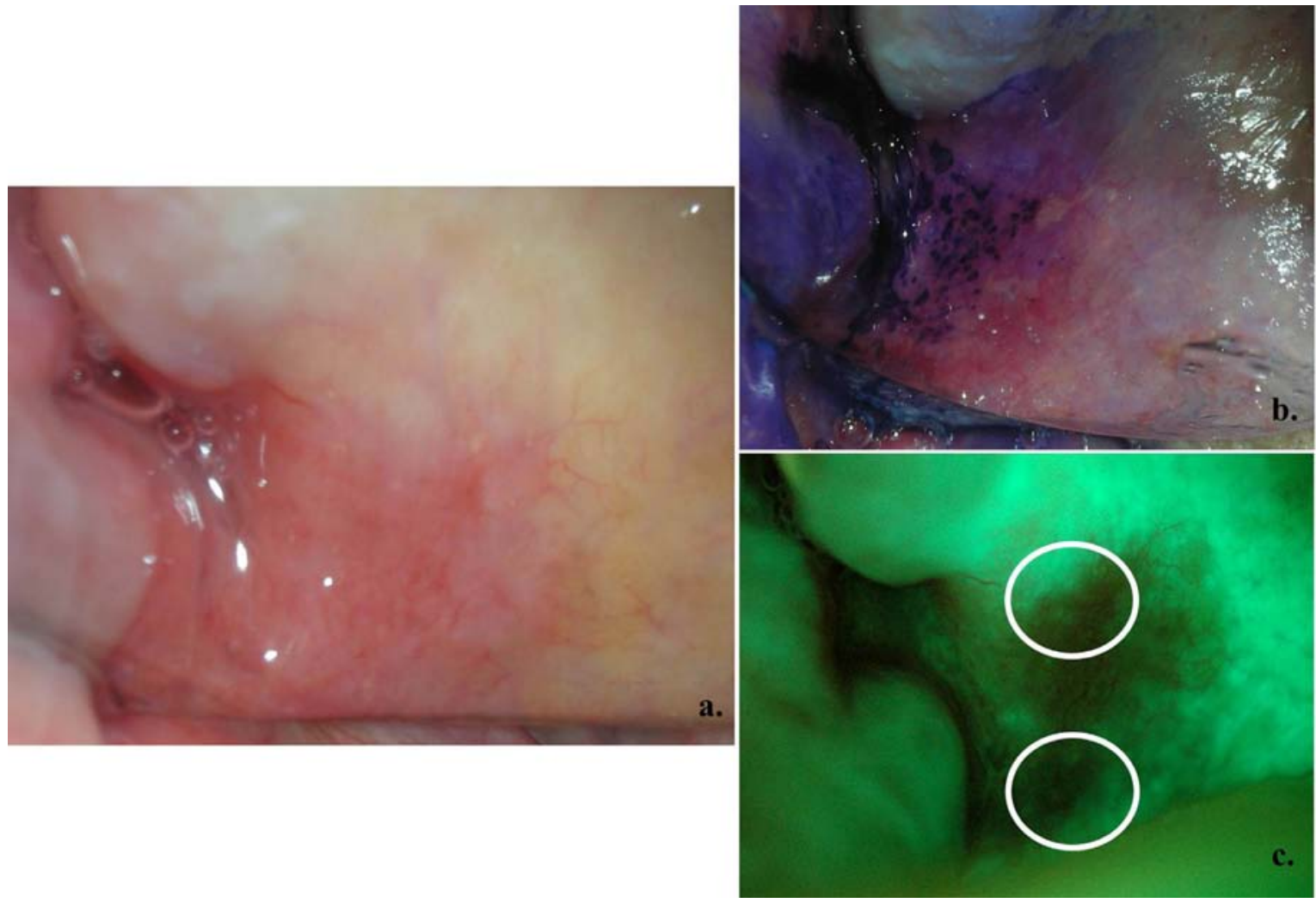

Fig. (4a-c). a) Red lesion on the right maxillary tuber is highlighted; the lesion is barely visible under conventional incandescent light; b) after TB staining, the lesion shows a punctuating uptake of the vital dye; c) the visualization of the lesion under fluorescent light shows an area of loss of fluorescence with a greater surface than the same lesion under conventional light. Two biopsies (white circle) have been performed, after using the fluorescent light, in order to better visualize lesions margins; the histopathological analysis showed the presence of squamous cell carcinoma infiltrating massively the surgical margins. (The color version of the figure is available in the electronic copy of the article). 
Table 1. The main non-invasive aids developed for oral cancer screening: indications and literature evidence; adapted from "Evidence-based clinical recommendations regarding screening for oral squamous cell carcinomas"- by Rethman M.P.-2010 [64]

\begin{tabular}{|c|c|c|}
\hline & Indication & Evidence \\
\hline Toluidine Blue & $\begin{array}{l}\text { May assist dental care providers, who } \\
\text { have advanced training in the clinical } \\
\text { assessment of potentially malignant disor- } \\
\text { ders and in the selection of the optimal } \\
\text { biopsy site. }\end{array}$ & $\begin{array}{l}\text { Insufficient evidence to recommend for or against the stand-alone use } \\
\text { of toluidine blue to enhance the identification of potentially malignant } \\
\text { lesions in the general population. }\end{array}$ \\
\hline $\begin{array}{l}\text { VELscope }^{\circledR} \\
\text { (LED Dental, Vancouver, British } \\
\text { Columbia, Canada) }\end{array}$ & $\begin{array}{l}\text { Could help identify potentially malignant } \\
\text { lesions that may not be visible to the } \\
\text { naked eye. }\end{array}$ & $\begin{array}{l}\text { There is some evidence that VELscope may improve the determina- } \\
\text { tion of surgical margins and selection of the optimal biopsy site; there } \\
\text { is insufficient evidence that these devices improve patient compliance } \\
\text { and that devices based on autofluorescence improve the detection of } \\
\text { potentially malignant lesions }\end{array}$ \\
\hline $\begin{array}{c}\text { ViziLite }^{\circledR} \\
\text { (Zila Pharmaceuticals, Fort Col- } \\
\text { lins, USA) } \\
\text { MicroLux }^{\circledR} \\
\text { (AdDent, Danbury, USA) }\end{array}$ & $\begin{array}{l}\text { Could enhance visual parameters of the } \\
\text { lesions in brightness, sharpness, surface } \\
\text { texture and size of lesion. }\end{array}$ & $\begin{array}{l}\text { There is insufficient evidence that devices based on tissue reflectance } \\
\text { improve the detection of potentially malignant lesions. }\end{array}$ \\
\hline $\begin{array}{l}\text { OralCDx }^{\circledR} \\
\text { (OralScan Laboratories, Inc., Suf- } \\
\text { fern, NY) }\end{array}$ & $\begin{array}{l}\text { May help the practitioner identify the } \\
\text { presence of atypical cells in mucosal } \\
\text { lesions. }\end{array}$ & $\begin{array}{l}\text { There is insufficient evidence to assess the validity of transepithelial } \\
\text { cytology of seemingly innocuous mucosal lesions. } \\
\text { There is evidence to use transepithelial cytology in case of suspicious } \\
\text { multiple mucosal lesions, in case of noncompliant patients during } \\
\text { their follow- up. }\end{array}$ \\
\hline $\begin{array}{l}\text { Narrowband imaging (Olympus } \\
\text { Optical Co. Ltd, Tokyo, Japan) }\end{array}$ & $\begin{array}{l}\text { May help the practitioner to enhance the } \\
\text { visualization of the mucosa surface and } \\
\text { microvasculature. }\end{array}$ & $\begin{array}{l}\text { There is no published evidence regarding the utility of narrowband } \\
\text { imaging in oral cancer detection. }\end{array}$ \\
\hline
\end{tabular}

moves only the cells of the superficial epithelial layers. The device is repeatedly brushed over the entire lesion by using moderate pressure in the same direction until the bleeding occurs, since it indicates that the lamina propria was reached and the sample is constituted by the whole epithelium. The obtained sample is then released in the middle third of a glass, fixed with a $100 \%$ ethanol solution, in order to allow both hematoxylin-eosin and Papanicolaou staining. This technique could be useful in patients with multiple lesions within the oral cavity: it is unlikely to obtain a representative biopsy specimen from multiple oral lesions in a patient without a previous history of OSCC. Similarly, this technique can be used in patients with lack of compliance or reluctant to undergo a further biopsy again during the follow-up of previously diagnosed lesions. It is well established that the identification of aneuploidy is perhaps the best predictive marker of possible neoplastic transformation [86].

It has been observed that the combination of cytological diagnosis and DNA-image cytometry may improve the overall sensitivity of the technique up to $100 \%$; this result may increase the application of this tool in early diagnosis of dysplasia [87, 88]. The main markers used in the cytological analysis are p53, Ki-67, DNA ploidy status, epigenetic changes (hypermethylation of the promoter region), and genomic instability, such as loss of heterozygosity (LOH) e microsatellite instability [89-92].

OralCDx ${ }^{\circledR}$ has been shown to have a sensitivity and specificity over $90 \%$ [93] and it has been proposed as an useful screening tool, in specific clinical situations, in case of clinically suspicious lesions, since it is considered as a simple, relatively inexpensive, highly sensitive and specific method [93-95].
The cytology is not comparable to the histological diagnosis and these two techniques should not be considered antithetical, but complementary: in case of abnormal cytology, it is strongly recommended that patient undergo a conventional biopsy [96].

\section{NARROWBAND IMAGING}

The NarrowBand Imaging- NBI (Olympus Optical Co. Ltd, Tokyo, Japan) is a novel endoscopic technique based on the use of special optical filters that narrow the light bandwidth to enhance the visualization of the mucosa surface and microvasculature [97, 98]. NBI is a method to visualize the structure of the surface capillaries, in particular the intrapapillary capillary loops, and the vascular architecture of the submucosa, allowing the physician to have additional information to early identify atypical tissues. In neoplastic lesions, blood vessels are modified by dilation, meandering and calibre irregularities distinguishable from healthy oral mucosa [99]. Their morphological changes are useful for early diagnosing cancers, determining the depth of invasion and the margin of resection [100].

The NBI system was first used by Gono et al, [101] and today is widely used for the examination of the esophageal and pharyngeal mucosa [102]. Since oral mucosa and esophagus are both covered by squamous epithelium presenting similar vascular architecture, it is likely that it could be powerful in detecting OSCC. In scientific literature only few case series have been reported in which the NBI system was used for early detection of precancerous lesions of the head and neck region, including pharynx and floor of the mouth [103]. Authors reported that the identification of the resection margin of the lesions thanks to NBI device was signifi- 
cantly improved when compared with other conventional systems, suggesting that this device may play an important role in the management of OSCC of the oral cavity [104]. NBI has several advantages as a diagnostic tool. The equipment is easy to manage. It is non-invasive and stress-free for the patient. It can be used for postoperative monitoring, for the PMDs follow-up and for establishing the lesion margin of resection. A recent study recommend a routine follow-up with NBI in patients with OSCC after treatment [105]. Since evidence is lacking, no firm conclusion can be drawn regarding the usefulness of NBI in early detection of oral cancer, but further studies are needed.

\section{CONCLUSIONS}

Early diagnosis of oral cancer is essential to ensure a better prognosis for patients in terms of disease-related morbidity and mortality. Screening by means of conventional clinical exam by using the incandescent light has been confirmed useful in detecting early and advanced oral cancer. However, the clinical features of the lesions alone cannot really allow distinguishing between benign and malignant lesions or selecting the potentially malignant lesions, which have a higher transformation risk. Up to now, oral biopsy is the only method to provide a definitive diagnosis in presence of oral suspicious lesions. Despite some limitations associated with the sampling site and the difficulty of classifying dysplasia, oral biopsy still represents the gold standard for OSCC and PMDs diagnosis. Furthermore, in presence of potentially malignant disorders, biopsy may allow distinguishing lesions at higher risk of transformation, but currently markers useful to detect oral lesions with inevitable risk of transformation are still lacking.

In a recent clinical trial conducted in India, where the prevalence of OSCC is very high, the Authors concluded that the conventional clinical exam couldn't lower the rate of disease-specific mortality in the general adult population. Considering this aspect, the Authors conclude that any procedure that facilitates visualization of suspicious lesions could help the clinician in its detection [106].

After reviewing the latest literature on the non-invasive methods for OSCC and PMDs diagnosis, there is insufficient evidence to recommend for or against the stand-alone use of these methods for identifying potentially malignant lesions.

Despite the lack of strong evidence that toluidine blue is a costeffective method of screening in the general population, toluidine blue can be used by clinicians experienced in oral medicine, thanks to its non-invasive nature and its capability to provide information regarding lesion margins and to guide the choice of biopsy site.

In the other reported studies, there is no evidence that devices based on tissue reflectance and autofluorescence can improve the detection of potentially malignant lesions. Some Authors have highlighted the possibility of early recognition of clinically occult lesions thanks to the direct visualization of tissue autofluorescence. However, these studies are only case series and therefore have a low level of evidence. The technique based on tissue autofluorescence provides an improvement in the visualization of some lesion clinical features, such as the margin extensions, and therefore, it should be considered as a complementary exam for the margin determination and selection of the optimal biopsy site in large or multifocal lesions or during surgery, by using oral biopsy as the "gold standard".

In a recent paper, tissue autofluorescence method reported relatively low values of sensitivity, indicating the probability of giving false negatives [107]. This is a great limitation in using this technique as screening tool in "high risk" population, since it can not always identify lesions with dysplasia, especially in case of mild dysplasia, which could represents the first histological change during oral carcinogenesis. However, specificity values are high, suggesting that the probability to give false positives is low.
Evidence in the literature to recommend the chemiluminescence method for early detection of oral cancer and potentially malignant disorders is strongly limited. Based on the collected data, the technique could be used just as an additional tool to improve clinical visualization, but only in case of white oral lesions. Moreover, although it has been reported that these light-based methods can provide an aid in the detection of malignant lesions, the limitation of conventional clinical examination in discerning the low-risk lesions from those at high risk of transformation is not completely resolved.

Brush cytology, and in particular OralCDx ${ }^{\circledR}$, has demonstrated validity as a method for identifying atypical cells disaggregated but, nevertheless, it must be emphasized that atypical findings can frequently be obtained if the test is performed on reactive or inflammatory lesions. If the brush test is performed on oral lesions that may mimic clinically potentially malignant disorders, without considering the patient's history and the clinical history of the lesion, it can lead to false positives. This method should not be considered an alternative exam to biopsy, rather there are some recommendations about the usefulness of the technique in some specific clinical situations. These may include patients that have multiple oral lesions without a history of oral cancer, or patients with poor compliance, which poorly accept the possibility of being subjected to several biopsies during the follow-up.

In conclusion, we should explain that "insufficient evidence" does not necessarily mean that the technique is effective or not is, but instead means that the literature review did not find sufficient evidence to support a recommendation of using these methods.

\section{CONFLICT OF INTEREST}

The authors confirm that this article content has no conflicts of interest.

\section{ACKNOWLEDGEMENT}

None declared.

\section{REFERENCES}

[1] Warnakulasuriya S. Global epidemiology of oral and oropharyngeal cancer. Oral Oncology 2009; 45(4-5): 309-16.

[2] Parkin DM, Bray F, Ferlay J, Pisani P. Global cancer statistics, 2002. Ca-A Cancer Journal for Clinicians 2005; 55(2): 74-108.

[3] Gillison ML. Current topics in the epidemiology of oral cavity and oropharyngeal cancers. Head and Neck 2007; 29(8): 779-92.

[4] Scott S, McGurk M, Grunfeld E. Patient delay for potentially malignant oral symptoms. European Journal of Oral Sciences 2008; 116(2): 141-7.

[5] Rogers SN, Brown JS, Woolgar JA, et al. Survival following primary surgery for oral cancer. Oral Oncology 2009; 45(3): 20111 .

[6] Hayat MJ, Howlader N, Reichman ME, Edwards BK. Cancer statistics, trends, and multiple primary cancer analyses from the surveillance, epidemiology, and end results (SEER) program. Oncologist 2007; 12(1): 20-37.

[7] Warnakulasuriya S, Johnson NW, Van Der Waal I. Nomenclature and classification of potentially malignant disorders of the oral mucosa. Journal of Oral Pathology and Medicine 2007; 36(10): 575-80.

[8] Böcking A, Sproll C, Stöcklein N, et al. Role of brush biopsy and DNA cytometry for prevention, diagnosis, therapy, and followup care of oral cancer. Journal of Oncology 2011.

[9] Saadeh CE. Chemotherapy- and radiotherapy-induced oral mucositis: review of preventive strategies and treatment. Pharmacotherapy 2005; 25(4): 540-54.

[10] Mignogna MD, Fedele S. Oral cancer screening: 5 Minutes to save a life. Lancet 2005; 365(9475): 1905-6.

[11] Patton LL. The effectiveness of community-based visual screening and utility of adjunctive diagnostic aids in the early detection of oral cancer. Oral Oncology 2003; 39(7): 708-23.

[12] Neville BW, Day TA. Oral cancer and precancerous lesions. Ca-A Cancer Journal for Clinicians 2002; 52(4): 195-215.

[13] Das BR, Nagpal JK. Understanding the biology of oral cancer. Medical Science Monitor 2002; 8(11): RA258-RA67. 
[14] Shugars DC, Patton LL. Detecting, diagnosing, and preventing oral cancer. The Nurse practitioner 1997; 22(6): 105, 9-10, 13-15 passim.

[15] Thomson PJ. Field change and oral cancer: New evidence for widespread carcinogenesis? International J Oral Maxillofacial Surgery 2002; 31(3): 262-6.

[16] Lee JJ, Hung HC, Cheng SJ, et al. Factors associated with underdiagnosis from incisional biopsy of oral leukoplakic lesions. Oral Surgery, Oral Medicine, Oral Pathology, Oral Radiology and Endodontology 2007; 104(2): 217-25.

[17] Seoane J, Varela-Centelles PI, Ramirez JR, Cameselle-Teijeiro J, Romero MA. Artefacts in oral incisional biopsies in general dental practice: a pathology audit. Oral Dis 2004; 10(2): 113-7.

[18] Seoane J, Varela-Centelles P, Ramirez JR, Romero MA, De La Cruz A. Artefacts produced by suture traction during incisional biopsy of oral lesions. Clinical Otolaryngology and Allied Sciences 2002; 27(6): 549-53.

[19] Patton LL, Epstein JB, Kerr AR. Adjunctive techniques for oral cancer examination and lesion diagnosis a systematic review of the literature. Journal of the American Dental Association 2008; 139(7): 896-905.

[20] Abbey LM, Kaugars GE, Gunsolley JC, et al. Intraexaminer and interexaminer reliability in the diagnosis of oral epithelial dysplasia. Oral Surgery, Oral Medicine, Oral Pathology, Oral Radiology and 1995; 80(2): 188-91.

[21] Fischer DJ, Epstein JB, Morton Jr TH, Schwartz SM. Reliability of histologic diagnosis of clinically normal intraoral tissue adjacent to clinically suspicious lesions in former upper aerodigestive tract cancer patients. Oral Oncology 2005; 41(5): 489-96.

[22] Du GF, Li CZ, Chen HZ, et al. Rose bengal staining in detection of oral precancerous and malignant lesions with colorimetric evaluation: A pilot study. International Journal of Cancer 2007; 120(9): 1958-63.

[23] Chen HZ. Rose bengal staining for clinical detection of oral premalignant lesions and carcinomas. Zhonghua kou qiang yi xue za zhi $=$ Zhonghua kouqiang yixue zazhi $=$ Chinese journal of stomatology 1992; 27(1): 44-7.

[24] Chen YW, Lin JS, Fong JHJ, et al. Use of methylene blue as a diagnostic aid in early detection of oral cancer and precancerous lesions. British Journal of Oral and Maxillofacial Surgery 2007; 45(7): 590-1.

[25] Chen YW, Lin JS, Wu CH, Lui MT, Kao SY, Fong Y. Application of in vivo stain of methylene blue as a diagnostic aid in the early detection and screening of oral squamous cell carcinoma and precancer lesions. Journal of the Chinese Medical Association 2007; 70(11): 497-503.

[26] Epstein JB, Scully C, Spinelli J. Toluidine blue and Lugol's iodine application in the assessment of oral malignant disease and lesions at risk of malignancy. Journal of Oral Pathology and Medicine 1992; 21(4): 160-3.

[27] Kurita H, Kamata T, Li X, Nakanishi Y, Shimane T, Koike T. Effectiveness of vital staining with iodine solution in reducing local recurrence after resection of dysplastic or malignant oral mucosa. Br J Oral Maxillofacial Surgery 2012; 50(2): 109-12.

[28] Petruzzi M, Lucchese A, Baldoni E, Grassi FR, Serpico R. Use of Lugol's iodine in oral cancer diagnosis: An overview. Oral Oncology 2010; 46(11): 811-3.

[29] Epstein JB, Guneri P. The adjunctive role of toluidine blue in detection of oral premalignant and malignant lesions. Curr Opin Otolaryngol Head Neck Surg 2009; 17(2): 79-87.

[30] Epstein JB, Oakley C, Millner A, Emerton S, Van Der Meij E, Le N. The utility of toluidine blue application as a diagnostic aid in patients previously treated for upper oropharyngeal carcinoma. Oral Surgery, Oral Medicine, Oral Pathology, Oral Radiology, Endodontics 1997; 83(5): 537-47.

[31] Missmann M, Jank S, Laimer K, Gassner R. A reason for the use of toluidine blue staining in the presurgical management of patients with oral squamous cell carcinomas. Oral Surgery, Oral Medicine, Oral Pathology, Oral Radiol Endodontol 2006; 102(6): 741-3.

[32] Epstein JB, Zhang L, Poh C, Nakamura H, Berean K, Rosin M. Increased allelic loss in toluidine blue-positive oral premalignant lesions. Oral Surgery, Oral Medicine, Oral Pathology, Oral Radiology, Endodontics 2003; 95(1): 45-50.

[33] Zhang L, Williams M, Poh CF, et al. Toluidine blue staining identifies high-risk primary oral premalignant lesions with poor outcome. Cancer Res 2005; 65(17): 8017-21.
[34] Epstein JB, Feldman R, Dolor RJ, Porter SR. The utility of tolonium chloride rinse in the diagnosis of recurrent or second primary cancers in patients with prior upper aerodigestive tract cancer. Head and Neck 2003; 25(11): 911-21.

[35] Guo Z, Yamaguchi K, Sanchez-Cespedes M, Westra WH, Koch WM, Sidransky D. Allelic losses in OraTest-directed biopsies of patients with prior upper aerodigestive tract malignancy. Clinical Cancer Research 2001; 7(7): 1963-8.

[36] Lingen MW, Kalmar JR, Karrison T, Speight PM. Critical evaluation of diagnostic aids for the detection of oral cancer. Oral Oncology 2008; 44(1): 10-22.

[37] Gupta A, Singh M, Ibrahim R, Mehrotra R. Utility of toluidine blue staining and brush biopsy in precancerous and cancerous oral lesions. Acta Cytologica 2007; 51(5): 788-94.

[38] Epstein JB, Güneri P. The adjunctive role of toluidine blue in detection of oral premalignant and malignant lesions. Current Opinion in Otolaryngology and Head and Neck Surgery 2009; 17(2): 79-87.

[39] Lonky NM, Edwards G. Comparison of chemiluminescent light versus incandescent light in the visualization of acetowhite epithelium. Am J Gynecol Health 1992; 6(1): 11-5.

[40] Suneja A, Mahishee, Agarwal N, Misra K. Comparision of magnified chemiluminescent examination with incandescent light examination and colposcopy for detection of cervical neoplasia. Indian J Cancer 1998; 35(2): 81-7.

[41] Wertlake PT, Francus K, Newkirk GR, Parham GP. Effectiveness of the Papanicolaou smear and speculoscopy as compared with the Papanicolaou smear alone: A community-based clinical trial. Obstetrics Gynecol 1997; 90(3): 421-7.

[42] Huber MA, Bsoul SA, Terezhalmy GT. Acetic acid wash and chemiluminescent illumination as an adjunct to conventional oral soft tissue examination for the detection of dysplasia: A pilot study. Quintessence International 2004; 35(5): 378-84.

[43] Nair DR, Pruthy R, Pawar U, Chaturvedi P. Oral cancer: Premalignant conditions and screening - An update. J Cancer Res Therapeut 2012; 8(SUPPL. 2): S57-S66.

[44] Fedele S. Diagnostic aids in the screening of oral cancer. Head neck Oncol 2009; 1: 5.

[45] Trullenque-Eriksson A, Muñoz-Corcuera M, Campo-Trapero J, Cano-Sánchez J, Bascones-Martínez A. Analysis of new diagnostic methods in suspicious lesions of the oral mucosa. Medicina Oral, Patologia Oraly Cirugia Bucal 2009; 14(5): E210-E6.

[46] Oh ES, Laskin DM. Efficacy of the ViziLite System in the Identification of Oral Lesions. Journal of Oral and Maxillofacial Surgery 2007; 65(3): 424-6.

[47] Ram S, Siar CH. Chemiluminescence as a diagnostic aid in the detection of oral cancer and potentially malignant epithelial lesions. International Journal of Oral and Maxillofacial Surgery 2005; 34(5): 521-7.

[48] Farah CS, McCullough MJ. A pilot case control study on the efficacy of acetic acid wash and chemiluminescent illumination (ViziLite, ${ }^{\mathrm{TM}}$ ) in the visualisation of oral mucosal white lesions. Oral Oncology 2007; 43(8): 820-4.

[49] Epstein JB, Silverman Jr S, Epstein JD, Lonky SA, Bride MA. Analysis of oral lesion biopsies identified and evaluated by visual examination, chemiluminescence and toluidine blue. Oral Oncology 2008; 44(6): 538-44.

[50] Awan KH, Morgan PR, Warnakulasuriya S. Utility of chemiluminescence (ViziLite ${ }^{\mathrm{TM}}$ ) in the detection of oral potentially malignant disorders and benign keratoses. J Oral Pathol Med 2011; 40(7): 541-4.

[51] Mehrotra R, Gupta DK. Exciting new advances in oral cancer diagnosis: Avenues to early detection. Head Neck Oncol 2011; $3(1)$.

[52] Awan KH, Morgan PR, Warnakulasuriya S. Evaluation of an autofluorescence based imaging system (VELscope, $®$ ) in the detection of oral potentially malignant disorders and benign keratoses. Oral Oncol 2011; 47(4): 274-7.

[53] McIntosh L, McCullough MJ, Farah CS. The assessment of diffused light illumination and acetic acid rinse (Microlux/DL $\left.{ }^{\mathrm{TM}}\right)$ in the visualisation of oral mucosal lesions. Oral Oncol 2009; 45(12): e227-e31.

[54] Alaa Rm M, Shibuya K, Fujiwara T, et al. Risk of lung cancer in patients with preinvasive bronchial lesions followed by autofluorescence bronchoscopy and chest computed tomography. Lung Cancer 2010 Oct 30. 
[55] De Veld DC, Witjes MJ, Sterenborg HJ, Roodenburg JL. The status of in vivo autofluorescence spectroscopy and imaging for oral oncology. Oral Oncol 2005; 41(2): 117-31.

[56] Lane PM, Gilhuly T, Whitehead P, et al. Simple device for the direct visualization of oral-cavity tissue fluorescence. J Biomed Opt 2006; 11(2): 024006.

[57] Ramanujam N, Mitchell MF, Mahadevan A, et al. Spectroscopic diagnosis of cervical intraepithelial neoplasia (CIN) in vivo using laser-induced fluorescence spectra at multiple excitation wavelengths. Lasers Surg Med 1996; 19(1): 63-74.

[58] Pavlova I, Williams M, El-Naggar A, Richards-Kortum R, Gillenwater A. Understanding the biological basis of autofluorescence imaging for oral cancer detection: high-resolution fluorescence microscopy in viable tissue. Clin Cancer Res 2008; 14(8): 2396-404.

[59] Ramanujam N. Fluorescence spectroscopy of neoplastic and nonneoplastic tissues. Neoplasia 2000; 2(1-2): 89-117.

[60] Jayaprakash V, Sullivan M, Merzianu M, et al. Autofluorescenceguided surveillance for oral cancer. Cancer Prev Res (Phila) 2009; 2(11): 966-74.

[61] Roblyer D, Kurachi C, Stepanek V, et al. Objective detection and delineation of oral neoplasia using autofluorescence imaging. Cancer Prev Res (Phila) 2009; 2(5): 423-31.

[62] Balevi B. Evidence-based decision making: should the general dentist adopt the use of the VELscope for routine screening for oral cancer? J Can Dent Assoc 2007; 73(7): 603-6.

[63] Trullenque-Eriksson A, Munoz-Corcuera M, Campo-Trapero J, Cano-Sanchez J, Bascones-Martinez A. Analysis of new diagnostic methods in suspicious lesions of the oral mucosa. Med Oral Patol Oral Cir Bucal 2009; 14(5): E210-6.

[64] Ingber DE. Cancer as a disease of epithelial-mesenchymal interactions and extracellular matrix regulation. Differentiation 2002; 70(9-10): 547-60.

[65] Arifler D, Pavlova I, Gillenwater A, Richards-Kortum R. Light scattering from collagen fiber networks: Micro-optical properties of normal and neoplastic stroma. Biophysical Journal 2007; 92(9): 3260-74.

[66] Lane PM, Gilhuly T, Whitehead P, et al. Simple device for the direct visualization of oral-cavity tissue fluorescence. Journal of Biomedical Optics 2006; 11(2).

[67] Awan KH, Morgan PR, Warnakulasuriya S. Evaluation of an autofluorescence based imaging system (VELscope) in the detection of oral potentially malignant disorders and benign keratoses. Oral Oncology 2011; 47(4): 274-7.

[68] Kois JC, Truelove E. Detecting oral cancer: A new technique and case reports. Dentistry Today 2006; 25(10): 94-7.

[69] Westra WH, Sidransky D. Fluorescence visualization in oral neoplasia: Shedding light on an old problem. Clinical Cancer Research 2006; 12(22): 6594-7.

[70] Moles DR, Downer MC, Speight PM. Meta-analysis of measures of performance reported in oral cancer and precancer screening studies. British Dental J 2002; 192(6): 340-4.

[71] Poh CF, Zhang L, Anderson DW, et al. Fluorescence visualization detection of field alterations in tumor margins of oral cancer patients. Clinical Cancer Res 2006; 12(22): 6716-22.

[72] Poh CF, Ng SP, Williams PM, et al. Direct fluorescence visualization of clinically occult high-risk oral premalignant disease using a simple hand-held device. Head and Neck 2007; 29(1): 71-6.

[73] Mehrotra R, Singh M, Thomas S, et al. A cross-sectional study evaluating chemiluminescence and autofluorescence in the detection of clinically innocuous precancerous and cancerous oral lesions. J Am Dental Association 2010; 141(2): 151-6.

[74] Ben BB. Evidence-based decision making: should the general dentist adopt the use of the VELscope for routine screening for oral cancer? CORD Conference Proceedings 2007; 73(7): 603-6.

[75] Poh CF, Zhang L, Anderson DW, et al. Fluorescence visualization detection of field alterations in tumor margins of oral cancer patients. Clin Cancer Res 2006; 12(22): 6716-22.

[76] Wang YC, Fang KH, Jung SM, Zheng J, Hao SP. Excisional biopsy with margin control for oral cancers. Head Neck 2010; 32(11): 1528-33.

[77] Gauthier P, Audet N, Guertin L, et al. Complete frozen section margins (with measurable 1 or $5 \mathrm{~mm}$ thick free margin) for cancer of the tongue: part 2: clinical experience. J Otolaryngol Head Neck Surg 2010; 39(1): 20-7.
[78] Balevi B. Assessing the usefulness of three adjunctive diagnostic devices for oral cancer screening: A probabilistic approach. Community Dentistry and Oral Epidemiology 2011;39(2): 171-6.

[79] López-Jornet P, De la Mano-Espinosa T. The efficacy of direct tissue fluorescence visualization in screening for oral premalignant lesions in general practice: an update. International journal of dental hygiene 2011; 9(2): 97-100.

[80] Paderni C, Compilato D, Carinci F, et al. Direct visualization of oral-cavity tissue fluorescence as novel aid for early oral cancer diagnosis and potentially malignant disorders monitoring. Int $\mathbf{J}$ Immunopathol Pharmacol 2011; 24(2(S)): 121-8.

[81] Hunter KD, Parkinson EK, Harrison PR. Profiling early head and neck cancer. Nature Reviews Cancer 2005; 5(2): 127-35.

[82] Mehrotra R, Singh MK, Pandya S, Singh M. The use of an oral brush biopsy without computer-assisted analysis in the evaluation of oral lesions: a study of 94 patients. Oral Surgery, Oral Medicine, Oral Pathology, Oral Radiology and Endodontology 2008; 106(2): 246-53.

[83] Queiroz JB, Lima CF, Burim RA, Brandao AAH, Cabral LAG, Almeida JD. Exfoliative cytology of the oral mucosa: Comparison of two collection methods. General dentistry 2010; 58(5): e196-e9.

[84] Navone R, Burlo P, Pich A, et al. The impact of liquid-based oral cytology on the diagnosis of oral squamous dysplasia and carcinoma. Cytopathology 2007; 18(6): 356-60.

[85] Divani S, Exarhou M, Theodorou LN, Georgantzis D, Skoulakis H. Advantages and difficulties of brush cytology in the identification of early oral cancer. Archive of Oncology 2009; 17(1-2): 11-2.

[86] Sudbø J, Kildal W, Risberg B, Koppang HS, Danielsen HE, Reith A. DNA content as a prognostic marker in patients with oral leukoplakia (Retraction in: New England Journal of Medicine (2006) 355: 18 (1927)). New Eng J Med 2001; 344(17): 1270-8.

[87] Poate TWJ, Buchanan JAG, Hodgson TA, et al. An audit of the efficacy of the oral brush biopsy technique in a specialist Oral Medicine unit. Oral Oncol 2004; 40(8): 829-34.

[88] Toyoshima T, Koch F, Kaemmerer P, Vairaktaris E, Al-Nawas B, Wagner W. Expression of cytokeratin 17 mRNA in oral squamous cell carcinoma cells obtained by brush biopsy: Preliminary results. J Oral Pathol Med 2009; 38(6): 530-4.

[89] Acha A, Ruesga MT, Rodrìguez MJ, Martìnez De Pancorbo MA, Aguirre JM. Applications of the oral scraped (exfoliative) cytology in oral cancer and precancer 2005; 10(2): 95-102.

[90] Remmerbach TW, Weidenbach H, Hemprich A, Böcking A. Earliest detection of oral cancer using non-invasive brush biopsy including DNA-image-cytometry: Report on four cases. Analytical Cellular Pathol 2003; 25(4): 159-66.

[91] Maraki D, Becker J, Boecking A. Cytologic and DNA-cytometric very early diagnosis of oral cancer. J Oral Pathol Med 2004; 33(7): 398-404.

[92] Zhang L, Rosin MP. Loss of heterozygosity: A potential tool in management of oral premalignant lesions? J Oral Pathol Med 2001; 30(9): 513-20.

[93] Scheifele C, Schmidt-Westhausen AM, Dietrich T, Reichart PA. The sensitivity and specificity of the OralCDx technique: Evaluation of 103 cases. Oral Oncol 2004; 40(8): 824-8.

[94] Hohlweg-Majert B, Deppe H, Metzger MC, et al. Sensitivity and specificity of oral brush biopsy. Cancer Investigation 2009; 27(3): 293-7.

[95] Felefli S, Flaitz CM. The oral brush biopsy: it's as easy as 1, 2, 3. Texas dental J 2000; 117(6): 20-4

[96] Eisen D, Frist S. Efficacy of the brush biopsy [1]. J Oral Maxillofacial Surgery 2003; 61(10): 1237.

[97] Kara MA, Ennahachi M, Fockens P, ten Kate FJW, Bergman JJGHM. Detection and classification of the mucosal and vascular patterns (mucosal morphology) in Barrett's esophagus by using narrow band imaging. Gastrointestinal Endoscopy 2006; 64(2): 155-66.

[98] Muto M, Minashi K, Yano T, et al. Early detection of superficial squamous cell carcinoma in the head and neck region and esophagus by narrow band imaging: A multicenter randomized controlled trial. J Clinical Oncol 2010; 28(9): 1566-72.

[99] Yang SW, Lee YS, Chang LC, Hwang CC, Luo CM, Chen TA. Use of endoscopy with narrow-band imaging system in evaluating oral leukoplakia. Head and Neck 2011.

[100] Muto M, Katada C, Sano Y, Yoshida S. Narrow band imaging: A new diagnostic approach to visualize angiogenesis in superficial 
neoplasia. Clinical Gastroenterology and Hepatology 2005; 3(7 SUPPL.): S16-S20.

[101] Gono K, Yamazaki K, Doguchi N, et al. Endoscopic Observation of Tissue by Narrowband Illumination. Optical Rev 2003; 10(4): 211-5.

[102] Ugumori T, Muto M, Hayashi R, Hayashi T, Kishimoto S. Prospective study of early detection of pharyngeal superficial carcinoma with the narrowband Imaging laryngoscope. Head and Neck 2009; 31(2): 189-94.

[103] Katada C, Nakayama M, Tanabe S, et al. Narrow band imaging for detecting superficial oral squamous cell carcinoma: A report of two cases. Laryngoscope 2007; 117(9): 1596-9.

Received: April 2, 2012
[104] Tan NCW, Mellor T, Brennan PA, Puxeddu R. Use of narrow band imaging guidance in the management of oral erythroplakia. Br J Oral Maxillofacial Surgery 2011; 49(6): 488-90.

[105] Chu PY, Tsai TL, Tai SK, Chang SY. Effectiveness of narrow band imaging in patients with oral squamous cell carcinoma after treatment. Head and Neck 2011.

[106] Sankaranarayanan R, Ramadas K, Thomas G, et al. Trivandrum Oral Cancer Screening Group. Effect of screening on oral cancer mortality in Kerala, India: a cluster-randomised controlled trial. Lancet 2005; 365(9475): 1927-1933

[107] Paderni C, Compilato D, Carinci F, et al. Direct visualization of oral cavity tissue fluorescence as novel aid for early oral cancer diagnosis and potentially malignant disorders monitoring. Int $\mathbf{J}$ Immunopathol Pharmacol 2011; 24: 201-10. 